NASA/CR-2002-211764

ICASE Report No. 2002-30

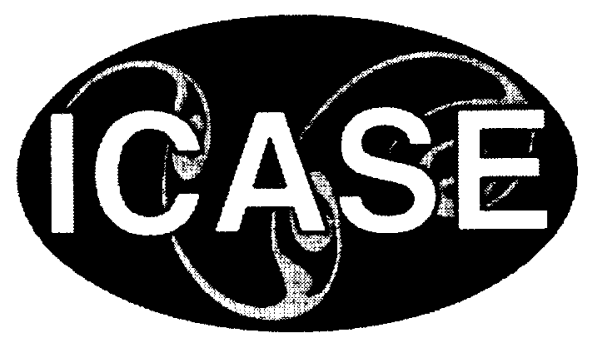

\title{
Backscattering and Nonparaxiality Arrest Collapse of Damped Nonlinear Waves
}

G. Fibich and B. Ilan

Tel Aviv University, Tel Aviv, Israel

S. Tsynkov

North Carolina State University, Raleigh, North Carolina and

Tel Aviv University, Tel Aviv, Israel 


\section{The NASA STI Program Office ... in Profile}

Since its founding, NASA has been dedicated to the advancement of aeronautics and space science. The NASA Scientific and Technical Information (STI) Program Office plays a key part in helping NASA maintain this important role.

The NASA STI Program Office is operated by Langley Research Center, the lead center for NASA's scientific and technical information. The NASA STI Program Office provides access to the NASA STI Database, the largest collection of aeronautical and space science STI in the world. The Program Office is also NASA's institutional mechanism for disseminating the results of its research and development activities. These results are published by NASA in the NASA STI Report Series, which includes the following report types:

- TECHNICAL PUBLICATION. Reports of completed research or a major significant phase of research that present the results of NASA programs and include extensive data or theoretical analysis. Includes compilations of significant scientific and technical data and information deemed to be of continuing reference value. NASA's counterpart of peer-reviewed formal professional papers, but having less stringent limitations on manuscript length and extent of graphic presentations.

- TECHNICAL MEMORANDUM. Scientific and technical findings that are preliminary or of specialized interest, e.g., quick release reports, working papers, and bibliographies that contain minimal annotation. Does not contain extensive analysis.

- CONTRACTOR REPORT. Scientific and technical findings by NASA-sponsored contractors and grantees.
- CONFERENCE PUBLICATIONS. Collected papers from scientific and technical conferences, symposia, seminars, or other meetings sponsored or cosponsored by NASA.

- SPECIAL PUBLICATION. Scientific, technical, or historical information from NASA programs, projects, and missions, often concerned with subjects having substantial public interest.

- TECHNICAL TRANSLATION. Englishlanguage translations of foreign scientific and technical material pertinent to NASA's mission.

Specialized services that complement the STI Program Office's diverse offerings include creating custom thesauri, building customized data bases, organizing and publishing research results . . . even providing videos.

For more information about the NASA STI Program Office, see the following:

- Access the NASA STI Program Home Page at http://www.sti.nasa.gov

- Email your question via the Internet to help@sti.nasa.gov

- Fax your question to the NASA STI Help Desk at (301) 621-0134

- Telephone the NASA STI Help Desk at (301) 621-0390

- Write to: NASA STI Help Desk NASA Center for AeroSpace Information 7121 Standard Drive Hanover, MD 21076-1320 
NASA/CR-2002-211764

ICASE Report No. 2002-30

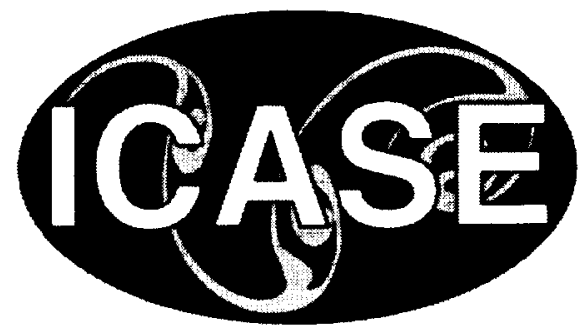

\section{Backscattering and Nonparaxiality Arrest Collapse of Damped Nonlinear Waves}

G. Fibich and B. Ilan

Tel Aviv University, Tel Aviv, Israel

S. Tsynkov

North Carolina State University, Raleigh, North Carolina and

Tel Aviv University, Tel Aviv, Israel

ICASE

NASA Langley Research Center

Hampton, Virginia

Operated by Universities Space Research Association 
Available from the following:

NASA Center for AeroSpace Information (CASI)

7121 Standard Drive

Hanover, MD 21076-1320

(301) $621-0390$
National Technical Information Service (NTIS)

5285 Port Royal Road

Springfield, VA 22161-2171

(703) 487-4650 


\title{
BACKSCATTERING AND NONPARAXIALITY ARREST COLLAPSE OF DAMPED NONLINEAR WAVES*
}

\author{
G. FIBICH ${ }^{\dagger+}, B, L_{A N}^{\dagger}$, ANI S. TSINKON+
}

\begin{abstract}
The critical nonlinear Schrödinger equation (NLS) models the propagation of intense laser light in Kerr media. This equation is derived from the more comprehessive nonlinear Helmholtz equation (NLH) by cmploying the paraxial approximation and neglecting the barkscattered waves. It is known that if the input power of the laser beam (i.e., $L_{2}$ norm of the initial solution) is sufficiently high, then the NLS model predicts that the beam will self-focus to a point (i.e., collap ie) at a finite propagation distance. Mathematically, this behavior corresponds to the formation of a singularity in the solution of the NLS. A key question which has been open for many years is whether the solution to the NLH, i.e., the "parent" equation. may nonetheless exist and remain regular everywhere, in particular for those initial conditions (input powers) that lead to blowup in the NLS. In the current study we address this que tion by introducing linear damping into both models and subsequently comparing the numerical solutions of the damped NLH (boundary-value problem) with the corresponding solutions of the damped NLS (initial-ralue problem). Linear damping is introduced in much the same way as done when analyzing the classical constant-coefficient Helmholtz equation using the limiting absorption principle. Numerically, we have found that it provides a very efficient tool for controlling the solutions of both the NLH and NLS. In partic alar, we have been able to identify initial conditions for which the NLS solution does become singular. whereas the NLH solution still remains regular everywhere. We believe that our finding of a larger domain of existence for the NLH than that for the NLS is accounted for by precisely those mechanisms that have been neglected when deriving the NLS from the NLH, i.e., nonparaxiality and backscattering.
\end{abstract}

Key words. Kerr medium, nonlinear wave propagation, self-focusing, singularity formation, linear damping, limiting absorption, two-way $\mathrm{ABCs}$

Subject classification. Applied and Numerical Mathematics

1. Introduction. The focusing critical nonlinear Schrödinger equa tion (NLS)

$$
i \psi_{z}(z, \boldsymbol{x})+\Delta_{\perp} \psi+|\psi|^{4 / d} \psi=0, \quad \psi(0, \boldsymbol{x})==\psi_{0}(\boldsymbol{x})
$$

where $x \in \mathbb{R}^{d}$ and $\Delta_{\perp}=\partial_{x_{1} x_{1}}+\cdots+\partial_{x_{d} x_{d}}$, arises in a variety of physiral contexts. Of foremost interest is the case $d=2$, which corresponds to the propagation of intense laser berms in Kerr media. In this case, $z$ is the axial coordinate in the direction of propagation, $x=(x, y)$ are the spatial coordinates in the transverse plane, $\Delta_{\perp}=\partial_{x x}+\partial_{y y}$ is the diffraction term (transverse Laplacian). and $|\psi|^{2} \psi$ describes the nonlinear polarization of the Kerr medium. It is well known that solutions to the critical NLS (1.1) can self-focus and eventually collapse, i.e., become singular, at a finite propagation distance, provided that their initial

\footnotetext{
*This research was supported by grant No. 2000311 from the (inited States-Isriel Binational Science Foundation (BSF). Jerusalem, Israel, and also by the National Aeronautics and Space Administration under NASA Contract No. NAS1 97046 while the third author was in residence at ICASF, NASA Langley Research Center, IIampton, VA 23681 2199, VSA.

${ }^{\dagger}$ School of Mathematical Sciences. Tel Aviv University, Ramat Aviv. Tel A iv 6!978, Israel.

${ }^{\ddagger}$ E-mail: fibich@math.tau.ac.il, URL: wwr.math.tau.ac.il/ fibich.

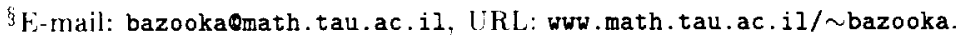

T Department of Mathematics, North Carolina State Lniversity, Box 8205, Raleigh. NC 27695. USA. Phone: (1-919)515-1877. Fax: (1-919)515-3798. E-mail: tsynkovomath.ncsu.edu, URL: www math.ncsu.edu/ $\sim$ stsynkov.
} 
power $N(0)=\int\left|\psi_{0}\right|^{2} d x$ exceeds a threshold power $N_{c}$, whose value depends only on the dimension $d[7,28]$. Since, however, physical quantities do not become infinite, and since in experiments laser beams continue to propagate beyond the NLS blowup point, the question arises as to what specific physical mechanism(s) among those that have been neglected when deriving the NLS from the Maxwell's equations, actually arrest(s) the collapse. We recall that the final stage in the derivation of the NLS is to disregard the backscattering and apply the paraxial approximation (see Section 2.2) to the critical nonlinear Helmholtz equation (NLH)

$$
\Delta E(z, x)+k_{0}^{2}\left(1+\epsilon|E|^{4 / d}\right) E=0, \quad \Delta \equiv \partial_{z z}+\Delta_{\perp},
$$

where $k_{0}$ is the linear wavenumber and the extent of nonlinearity is measured by the quantity $\epsilon=4 \epsilon_{0} c n_{2}$, where $n_{2}$ is the Kerr coefficient, see, e.g., $[3,19]$. Therefore, it is natural to ask whether going back from the NLS to the NLH, i.e., adding nonparaxiality and backscattering, is sufficient to guarantee existence of the solution with no singularities. In other words, for a given initial condition that leads to blowup in the critical NLS, does the NLH (always) have a solution that remains regular everywhere?

The foregoing question has been open for many years. In his celebrated 1965 paper in Physical Review Letters [15], which was the first publication in the literature to predict that the solutions to the critical NLS can become singular, Kelley was careful to note that the paraxial approximation, and hence the entire NLS model, breaks down near the singularity. Feit and Fleck [4] were the first to demonstrate that nonparaxiality of the beam can arrest the blowup, by showing numerically that the initial conditions that lead to singularity formation in the NLS, result in focusing-defocusing oscillations in the NLH. In these simulations, however, they did not solve a true boundary-value problem for the NLH. Instead, they solved an initial-value problem for a "modified" NLH that only describes the right-propagating wave (while introducing several additional assumptions along the way). Akhmediev and collaborators $[1,2]$ analyzed an initial-value problem for a different "modified" NLH; their numerical simulations also suggested that nonparaxiality arrests the singularity formation. Both numerical approaches [4] and [1,2], however, did not account for the effect of backscattering. Fibich [5] applied asymptotic analysis to derive an ODE in $z$ for self-focusing in the presence of small nonparaxiality. His analysis suggests that nonparaxiality indeed arrests the singularity formation, resulting instead in decaying focusing-defocusing oscillations. However, backscattering effects were neglected in this asymptotic analysis.

The aforementioned studies $[1,2,4,5,15]$ have prompted a general belief that nonparaxiality arrests the collapse. However, no rigorous proof of global existence for the NLH has ever been provided. Moreover, all the simulations in the above studies neglected the backscattering and considered only the forward-propagating field. The first numerical solutions of the NLH as a true boundary value problem, with backscattering effects fully included, have been obtained by Fibich and Tsynkov in [12], using a high-order discretization supplemented by a new two-way artificial boundary condition (ABC). The simulations in [12] were performed for the values of the input power of up to $90 \%$ of the threshold $N_{\mathrm{c}}$, and they have captured the mild selffocusing of the corresponding solutions. In a subsequent paper [10], we have corroborated experimentally the prediction of the asymptotic analysis that the magnitude of the backscattered signal scales quadratically with the nonparaxiality parameter $f$ (see Section 2.2), and that the computed NLH solutions converge to the corresponding NLS solutions as $f$ goes to zero.

The numerical methodology of [12] was obviously not free of limitations of its own. Foremost, we could not obtain converging solutions for initial powers equal to or higher than the critical value $N_{c}$. In [12], we have considered initial powers of only up to $90 \%$ of $N_{c}$; in the current paper we computed the NLH solutions for up to $N(0)=0.99 N_{c}$ (see Section 4). In the course of these simulations we have noticed that as $N(0)$ 
approaches the critical power from below, the convergence rate of the iterations slows down noticeably: This makes the simulations for higher subcritical values of $N(0)\left(0.99 N_{c}<N(0)<N_{c}\right)$ difficult to conduct, although it is reasonable to assume that the NLH solution will converge for input powers all the way up to $N_{c}$. However, for the input power $N(0)$ exactly equal to $N_{c}$ the convergence of nonlinear iterations of [12] is lost (see Section 4).

The aforementioned slowdown of convergence for input powers slightly below $N_{c}$ should be attributed to either deficiencies of the method, or to insufficient computational resources, or to both. As concerns the iteration method of [12] itself, it is the most straightforward approach based on simply freezing the nonlinearity; most likely, it can be improved or replaced by a more acivanced technique, and we plan on looking into this issuc in the future. As for the computer resources requirements, they are determined by the size of the computational domain, which should be sufficiently large so that to meet the condition of nearlinear propagation in the far field, see [12]; and by the grid size, which should be sufficiently fine to resolve a given wave length and the sharp near-blowup profile. These requirements become more stringent for higher input powers, which decay at larger distances and/or undergo stronger focusing. In other words, the higher the input power the larger the domain and/or the finer the grid that one seeds to use in order to maint ain the same solution quality and/or convergence rate. In our previous simulations wo have, indeed, seen examples of diverging NLH solutions with suberitical input powers which converged on a larger computational domain and/or at a finer resolution. It is still unclear, however, whether having more computer resources and/or a better nonlinear iteration scheme will allow one to solve the NLH for initial conditions that lead to collapse in the NLS, or whether the convergence breakdown at $N(0)>N_{\mathrm{c}}$ is an indication of the loss of solvability of the NLH, or loss of regularity of the solution.

As such, in the current paper we explore an alternative approach o the issue of solving the NLH in the blowup regime of the NLS, by considering the linearly damped NLH and the corresponding linearly damped NLS. The addition of linear damping is not an ad hoc procedur". Indeed, an electromagnetic wave is always partially absorbed by the medium through which it propagates, an effect neglected in either the original undamped NLH or NLS, both of which model the propagation under "ideal transparency." A mathematical motivation to add linear damping comes from the so-ralle limiting absorption principle that is used for identifying the unique solutions of the linear Helmholtz equation, see, e.g., [27]. It is known that the classical constant-coefficient homogeneous Helmholtz equation

$$
\Delta E+k_{0}^{2} E=0
$$

has non-trivial solutions on the entire space even in the class of functions that vanish at infinity, which obviously amounts to non-uniqueness. To fix the problem, the additional Sommerfeld boundary conditions need to be introduced at infinity that basically distinguish between the incoming and outgoing waves. On the other hand, when a complex absorption coefficient is added, the new damped equation

$$
\Delta E+k_{0}^{2}(1+i \delta) E=0
$$

has only trivial solution. Consequently, its inhomogeneous counterpar will be uniquely solvable for any compactly-supported right-hand side in rather wide classes of functions, such as tempered distributions. see [27]. Moreover, when $\delta \longrightarrow \pm 0$, the unique solution of the inhomogennous damped equation will converge uniformly on the entire space to the solution of the respective undaniped equation that corresponds to either the radiation of waves toward infinity (outgoing waves), or conversely, the incidence of waves from infinity (incoming waves), where the distinction is rendered by the sign of $\delta$. This, in particular, implies that if 
we decide to keep a small but finite damping in the equation, we may expect its solution to be uniformly close to the solution of the undamped equation that is driven by the same source terms and is composed of either only outgoing or only incoming waves in the far field. The latter consideration is especially important in the context of our iteration algorithm, see Section 3 and [12] for detail, which basically reduces to a repeated solution of the constant-coefficient Helmholtz equation driven by a variety of compactly supported right-hand sides and subject to the radiation boundary conditions in the far field.

Solving the damped NLH numerically as a true boundary value problem required only minor changes in the algorithm of [12] for the undamped NLH, which are described in Section 3. At the same time, the addition of damping allows us to better control the solution. In particular, damping decreases the solution magnitude in the far field, which is a key requirement for the validity of the artificial boundary conditions $(\mathrm{ABCs})$ of [12]. As a result, we have been able to consider initial conditions with the powers well above $N_{c}$.

Let us recall that for a given initial condition that leads to the blowup in the undamped critical NLS, there is a threshold value $\delta_{\mathrm{th}}^{\mathrm{S}}$ of the damping parameter $\delta$ such that if $\delta>\delta_{\mathrm{th}}^{\mathrm{S}}$ then linear damping arrests the collapse, whereas when $\delta<\delta_{\mathrm{th}}^{\mathrm{s}}$ the solution of the NLS blows up, see [6]. ${ }^{1}$ In the numerical simulations of the damped NLH reported hereafter we found a similar threshold value $\delta_{\mathrm{th}}^{\mathrm{H}}$ such that for $\delta>\delta_{\mathrm{th}}^{\mathrm{HI}}$ the solution exists and is regular everywhere, whereas when $\delta<\delta_{\text {th }}^{\mathrm{H}}$ the iteration scheme diverges. As has been mentioned, in the latter case it is not clear whether the divergence indicates that there is no solution to the NLH, or that our computational resources are insufficient (or the iteration scheme is suboptimal) to calculate the solution. Therefore, we can conclude that the actual (analytical) threshold value $\hat{\delta}_{\mathrm{th}}^{\mathrm{H}}$, such that regular solutions to the NLH exist for all $\delta>\hat{\delta}_{\mathrm{th}}^{\mathrm{H}}$, is less or equal than the computed threshold $\delta_{\mathrm{th}}^{\mathrm{H}}$, which is determined from the simulations, i.e., that $0 \leq \hat{\delta}_{\mathrm{th}}^{\mathrm{H}} \leq \delta_{\mathrm{th}}^{\mathrm{H}}$.

The main result of the current study is that

$$
\delta_{\mathrm{th}}^{\mathrm{H}}<\delta_{\mathrm{th}}^{\mathrm{S}} .
$$

In other words, for a given initial condition that leads to the blowup in the undamped NLS, there is an entire range of values for the damping coefficient: $\delta_{\mathrm{th}}^{\mathrm{H}}<\delta<\delta_{\mathrm{th}}^{\mathrm{s}}$, for which the damped NLS solution will blow up, but the NLH solution will be regular everywhere. Therefore, we can conclude that nonparaxiality and backscattering arrest the collapse when the damping parameter is in the range $\delta_{\mathrm{th}}^{\mathrm{H}}<\delta<\delta_{\mathrm{th}}^{\mathrm{S}}$. Whether NLH solutions exist for infinitely small linear damping as well, i.e., in the limit $\delta \longrightarrow 0$, is the question that yet remains to be answered. We believe, however, that this question should be considerably easier to address, both numerically and analytically, than the question of solvability of the original undamped NLH.

\section{Formulation of the Problem.}

2.1. The Nonlinear Helmholtz Equation. A typical setup for the propagation of electromagnetic waves in a Kerr medium is shown in Figure 2.1. An inconing laser beam with known characteristics impinges normally on the planar interface $z=0$ between the linear and the nonlinear medium. The electric field $E=$ $E(z, x)$ is governed by the nonlinear Helmholtz equation (1.2). For simplicity, we consider the cylindricallysymmetric case ${ }^{2}$ where $E=E(z, r)$ and $r=\sqrt{x_{1}^{2}+\ldots+x_{d}^{2}}$. The nonlinear medium occupies the semi-space $z \geq 0$ (see Figure 2.1). Consequently, the NLH (1.2) has to be supplemented by boundary conditions at $z=0$

\footnotetext{
${ }^{1}$ Self-focusing in the critical NLS is highly sensitive to the effect of small perturbations. Some perturbations can arrest the collapse even if they are initially infinitesimally small [11]. In contrast, an infinitesimally small linear damping does not arrest the collapse, and its sufficient amount must be present to regularize the solution.

${ }^{2} \mathrm{This}$ assumption is quite reasonable, since even when the initial conditions of the NLS are not cylindrically-symmetric, near the singularity the solution becomes cylindrically-symmetric [8].
} 


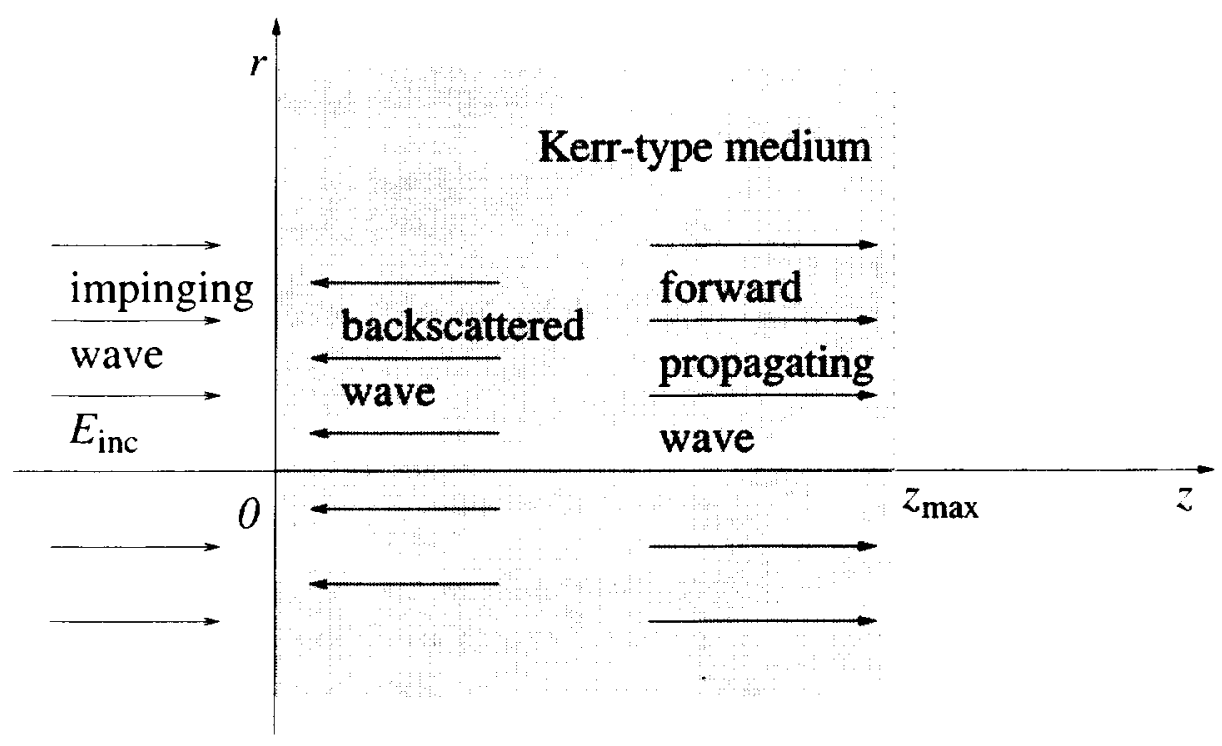

FIG. 2.1. Schematic of propagation of waves in Kerr media.

and $z \longrightarrow+\infty$. We require that as $z \longrightarrow+\infty, E$ have no left-traveling components and that the propagation be diffraction-dominated with the field amplitude decaying to zero, i.e., $\lim _{z \rightarrow \infty} \max _{0 \leq r<\infty}|E(z, r)|=0$, which also means that the nonlinear wavenumber $k^{2} \equiv k_{0}^{2}\left(1+\epsilon|E|^{4 / d}\right)$ approaches its linear limit: $\lim _{i \rightarrow+\infty} k^{2}=k_{0}^{2}$. In other words, at large $z$ 's the solution should be a linear superposition of right-traveling waves. Since the actual numerical simulation is carried out on a truncated domain $0 \leq z \leq z_{\max }$ (Figure 2.1), the desired behavior of the solution as $z \longrightarrow+\infty$ has to be captured by a far-field artificial boundary condition (ABC) at the artificial boundary $z=z_{\max }$. This boundary condition should guarantee a reflectionless propagation of all the waves traveling towards $z=+\infty$. Often, boundary conditions designed to ensure the transparency of the outer boundary to the outgoing waves are called radiation boundary conditions [24].

The situation is more complex at the interface $z=0$, where the total field $E(0, r)$ is composed of a given incoming (right-traveling) component $E_{\text {inc }}(0, r)$ and an unknown backs attered (left-traveling) component $E_{\text {scal }}(0, r)$, i.e.,

$$
E(0, r)=E_{\text {inc }}(0, r)+E_{\text {scat }}(0, r) .
$$

As such, the boundary condition at $z=0$ has to guarantee the reflictionless propagation of any lefttraveling wave through the interface and at the same time be able to correctly prescribe the incoming signal. Implementation of such a two-way $A B C$ was first carried out in [12] for the undamped NLS, and is extended to the damped case in Section 3.3.

Finally, the electric field vanishes as $r \longrightarrow+\infty$. In practice, we trun cate the domain at some large but finite $r_{\max }$ and require that $E\left(z, r_{\max }\right)=0$.

2.2. Paraxial Approximation and the Nonlinear Schrödinger Equation. We first introduce the dimensionless quantities $\tilde{r}, \tilde{z}$, and $\psi$ as

$$
\left.\tilde{r}=\frac{r}{r_{0}}, \quad \tilde{z}=\frac{z}{2 L_{D F}}, \quad E=e^{i k_{0} z}\left(\epsilon r_{0}^{2} k_{0}^{2}\right)^{-d / 1}\right)(z, r),
$$


where $r_{0}$ is the transverse width of the input beam and $L_{D F}=k_{0} r_{0}^{2}$ is the diffraction length. Then, by substituting tho quantities (2.1) into the NLH (1.2) and dropping the tildes, we obtain

$$
i \psi_{z}+\Delta_{\perp} \psi+|\psi|^{4 / d} \psi=-4 f^{2} \psi_{z z}
$$

where $f=1 / r_{0} k_{0}=\lambda / 2 \pi r_{0}$ is the nonparaxiality parameter.

The standard derivation of the NLS is motivated by the observation that $f \ll 1$, since typically $\lambda \ll r_{0}$. This suggests that one can neglect the $\psi_{z z}$ term, i.e., apply the paraxial approximation, and obtain the nonlinear Schrödinger equation

$$
i \psi_{z}(z, r)+\Delta_{\perp} \psi+|\psi|^{4 / d} \psi=0
$$

which is the same as the previously introduced equation (1.1), except that in (2.3) we use $r$ instead of $x$ for simplicity. The NLS (2.3) is supplemented by the initial condition at $z=0$ :

$$
\psi(0, r)=\left(\epsilon r_{0}^{2} k_{0}^{2}\right)^{d / 4} E_{\mathrm{inc}}(0, r)
$$

Subsequently, it needs to be integrated by a "time"-marching algorithm, where the direction of propagation $z$ plays the role of time. We reemphasize that backscattering effects are not taken into account by the NLS (2.3). Indeed, once (2.3) is solved, the overall solution, according to (2.1), is the slowly varying amplitude times the forward propagating oscillatory component $e^{i k_{0} z}$.

2.3. Linear Damping. When damping, i.e., linear absorption, is included, the NLH (1.2) becomes

$$
\Delta E(z, x)+k_{0}^{2}\left(1+i \delta+\epsilon|E|^{4 / d}\right) E=0,
$$

where $k_{0}$ is the (real part of the) wavenumber,

$$
\delta=\frac{\Im\left(n_{0}^{2}\right)}{\Re\left(n_{0}^{2}\right)},
$$

and $n_{0}$ is the linear index of refraction of the medium. The corresponding NLS (2.3) becomes (see eq. (2.1))

$$
i \psi_{z}+\Delta_{\perp} \psi+|\psi|^{4 / d} \psi+i r_{0}^{2} k_{0}^{2} \delta \psi=0
$$

By definition, optical transparency of the medium means that the damping is small. For example, for water in the visible regime [14],

$$
\frac{\Im\left(n_{0}^{2}\right)}{\Re\left(n_{0}^{2}\right)} \sim 10^{-7}
$$

Having small physical values of damping also agrees well with the mathematical reasoning behind the limiting absorption principle. As indicated in Section 1 (see, e.g., [27] for detail), for a classical constant-coefficient Helmholtz operator of (1.3a), the introduction of a small complex absorption coefficient of the appropriate sign [as in (1.3b)] implies that there will be a unique solution for any compactly supported excitation, and that this solution will be uniformly close in the entire space $\mathbb{R}^{d+1}$ to the solution of the corresponding undamped linear Helmholtz equation driven by the same sources and subject to the radiation boundary conditions in the far field. In the following Section 3 , we show that for the formulation analyzed in this paper the proper sign of $\delta$ is positive.

As we have noted before, the physical case that corresponds to the propagation of laser beams in bulk Kerr media is $d=2$. However, in order to reduce the complexity of the computations we rather consider a 
simpler case $d=1$, as was previously done in [12]. Thus, the damped NI.H for $E=E(z, r)$ and the damped NLS for $\psi=\psi(z, r)$ that are solved numerically in this study are

$$
E_{z z}(z, r)+E_{r r}+k_{0}^{2}\left(1+i \delta+\epsilon|E|^{1}\right) E=0
$$

and

$$
i \psi_{*}(z, r)+\psi_{r r}+i r_{0}^{2} k_{0}^{2} \delta \psi+\left|\psi^{4}\right|^{4} \vartheta^{\prime}=0 .
$$

respectively.

3. Numerical Methods. The damped NLH (2.6) is solved using fourth-order finite differences. The methodology of solution is outlined below in Section 3.1; it is similar to the one that we have introduced in our previous work [12] for solving the undamped NLH. The choice of a higher-order method is motivated primarily by the necessity to resolve a small-scale phenomenon of backscattering at the background of the forward propagating waves. The damped NLS (2.7) is also solved by a fourth-order scheme; it is natural to expect that this will leave less room for potential purely numerical discrepancies between the two techniques and as such, will allow for a more accurate comparison. Besides, it is generally known that higher-order methods provide for a better resolution of waves.

3.1. Discretization of the NLH and Solution Methodology. We use a conventional fourth-order central-difference discretization for the Laplacian $\Delta=\partial_{z z}+\partial_{r r}$ of (2.6) in so doing the stencil is five-node wide in each coordinate direction. As the equation is nonlinear, we implement a nested iteration scheme. On the outer loop, we freeze the nonlinearity, i.e., consider the coefficient $k^{2} \equiv k_{0}^{2}\left(1+i \delta+\epsilon|E|^{4}\right)$ as a given function of the coordinates $z$ and $r$, which is actually obtained by taking the quantity $|E|^{4}$ from the previous iteration, see (2.6). This way we arrive at a linear equation wits variable coefficients. The latter is also solved by iterations on the inner loop of the nested scheme. Here, we leave the entire varying part of the equation, which is proportional to $\epsilon$, on the lower level, and on the upper level need to invert only the constant-coefficient linear damped Helmholtz operator $\Delta+k_{0}^{2}(1+i \delta) I$ (cf. equation (1.3b))).

Formally; our iteration scheme resembles the fixed-point approach. however, no rigorous convergence theory is available yet, and the convergence is assessed experimentally. The advantage of using these nested iterations is that first, the method eventually reduces to the repeated solution of one and the same linear constant coefficient equation driven by different source terms, which can be done efficiently at the discrete level. Second, the radiation boundary conditions at $z=z_{\max }$ and the two-way ABCs at $z=0$, see Figure 2.1, are most convenient to set on the upper time level of the iteration scheme already for the linear constantcoefficient operator.

To solve the linear constant-coefficient damped discrete Helmholtz equation

$$
\Delta^{(h)} E+k_{0}^{2}(1+i \delta) E=g,
$$

where $g$ is the right-hand side generated on the previous iteration. we first separate the variables by implementing the discrete Fourier transform in the transverse direction $r$; the boundary conditions are symmetry at $r=0$ and zero Dirichlet at $r=r_{\max }$ (see Section 2.1). This yields a collection of fourth-order one-dimensional finite-difference equations (grid index $n$ corresponds to the continuous variable $z$ ):

$$
\frac{-\hat{E}_{n-2}+16 \hat{E}_{n-1}-30 \hat{E}_{n}+16 \hat{E}_{n+1}-\hat{E}_{n+2}}{12 h_{z}^{2}}+\left(k_{0}^{2}(1+i \delta)-\lambda_{m}\right) \hat{E}_{n}=\hat{g}_{n}
$$


parameterized by the dual Fourier variable $\lambda_{m}$, the latter is defined by formula (29) of [12]. Each equation (3.2) needs to be solved independently. ${ }^{3}$ The two-way and radiation $\mathrm{ABCs}$ at $z=0$ and $z=z_{\max }$, respectively, for the discrete equation (3.1) are set in the Fourier space, i.e., individually for each onedimensional equation (3.2). This is done by first identifying the linearly-independent eigen-modes for the homogeneous version of this equation. It is important to note that even though the original differential equation is of the second order, we are using its fourth-order approximation and as such, each homogeneous discrete one-dimensional equation of type (3.2) has four linearly independent solutions. These solutions are $q_{1}^{n}, q_{1}^{-n}, q_{2}^{n}$, and $q_{2}^{-n}$, see [12], where $q_{1}, 1 / q_{1}, q_{2}$, and $1 / q_{2}$ are roots of the characteristic algebraic equation

$$
-1+16 q+\left(12 h_{z}^{2}\left(k_{0}^{2}(1+i \delta)-\lambda_{m}\right)-30\right) q^{2}+16 q^{3}-q^{4}=0 .
$$

3.2. Roots of the Characteristic Equation. It is indeed easy to see that equation (3.3) has two pairs of mutually inverse roots. We first notice that this equation originates from a central-difference, i.e., symmetric, discretization (3.2). As such, if $q$ is a root, then $q^{-1}$ is obviously a root as well, which can be verified by direct substitution. Then, to actually find the roots we rewrite the polynomial on the left-hand side of (3.3) as

$$
\begin{aligned}
& \left(q-q_{1}\right)\left(q-q_{1}^{-1}\right)\left(q-q_{2}\right)\left(q-q_{2}^{-1}\right) \\
\equiv & -1+\left(d_{1}+d_{2}\right) q-\left(2+d_{1} d_{2}\right) q^{2}+\left(d_{1}+d_{2}\right) q^{3}-q^{4},
\end{aligned}
$$

where

$$
d_{1}=q_{1}+q_{1}^{-1}, \quad d_{2}=q_{2}+q_{2}^{-1},
$$

and match the coefficients. In so doing, we obtain

$$
d_{1}+d_{2}=16, \quad-2-d_{1} d_{2}=12 h_{z}^{2}\left(k_{0}^{2}(1+i \delta)-\lambda_{m}\right)-30,
$$

so that each pair of roots: $q_{1}, q_{1}^{-1}$ and $q_{2}, q_{2}^{-1}$, can be found by solving the corresponding quadratic equation:

$$
q^{2}-d_{1} q+1=0
$$

or

$$
q^{2}-d_{2} q+1=0
$$

while the coefficients $d_{1}$ and $d_{2}$ are, in turn, determined by solving quadratic equations (3.4).

At this stage, the key difference between the current analysis for the damped equation and the previous analysis for the undamped equation of [12] needs to be emphasized. As shown in [12], when $\delta=0$ the first pair of solutions of the homogeneous equation (3.2), $q_{1}^{n}$ and $q_{1}^{-n}$, approximates the genuine "longitudinal," i.e., $z$-aligned, modes of the undamped homogeneous differential equation (1.3a):

$$
\hat{E}_{1}=e^{i k_{c} z}, \quad \text { and } \quad \hat{E}_{2}=e^{-i k_{c} z},
$$

respectively. The functions $\hat{E}_{1}=\hat{E}_{1}(z)$ and $\hat{E}_{2}=\hat{E}_{2}(z)$ are two linearly-independent solutions of the ODE

$$
\hat{E}_{z z}+\left(k_{0}^{2}-\lambda\right) \hat{E}=0
$$

\footnotetext{
${ }^{3}$ Note, the discrete equations (3.1) and (3.2) are very similar to the corresponding discrete equations studied in [12] except. that previously we had no damping.
} 
obtained by Fourier transforming equation (1.3a) with respect to $r ; \lambda$ is the dual variable. In formulae (3.6), we have denoted $k_{f}=\sqrt{k_{0}^{2}-\lambda}$, and a particular branch of the square root that we always take is $\sqrt{\rho e^{i \theta}}=\rho^{1 / 2} e^{i \theta / 2}$. The two continuous modes (3.6) may be either traveling or evanescent waves depending on whether the real quantity $k_{c}^{2}=\left(k_{0}^{2}-\lambda\right)$ is positive or negative, or in of her words, whether the dual Fourier variable $\lambda$ is less or greater than $k_{0}^{2}$. To demonstrate the aforementior ed approximation property for the undamped $(\delta=0)$ discretization (3.2), we re-define $k_{c}=\sqrt{k_{0}^{2}-\lambda_{n}}$, introduce $\alpha=h_{z}^{2} k_{r}^{2}$, and show in [12] that if $\alpha>0$ then $q_{1}$ and $q_{1}^{-1}$ are complex conjugate roots of the charateristic equation (3.3). Both these roots have unit magnitude: $\left|q_{1}\right|=\left|q_{1}^{-1}\right|=1$, which indicates that $q_{1}^{\prime \prime}$ and $q_{1}^{-n}$ are pure discrete traveling waves. Moreover, if $\alpha \ll 1$ then (see [12])

$$
q_{1}=e^{i k_{c} h_{z}}+\mathcal{O}\left(\left(k_{c} h_{z}\right)^{5}\right), \quad q_{1}^{-1}=e^{-i k_{c} h_{z}}+\mathcal{O}\left(\left(k_{c} h_{z}\right)^{5}\right)
$$

Equalities (3.8) imply that in the undamped case $\delta=0, q_{1}^{n}$ is a discrete counterpart of the right-traveling wave $\hat{E}_{1}$, and $q_{1}^{-n}$ is a discrete counterpart of the left-traveling wave $\hat{E}_{2}$ : the approximation is obviously fourth-order accurate because on the grid $z_{n}=h_{z} n$. If $\alpha<0$ and still $\delta:=0$, then we again show in [12] that $\left|q_{1}\right|<1$ and $\left|q_{1}^{-1}\right|>1$, which indicates that $q_{1}^{n}$ is a right-evanescent waw and $q_{1}^{-n}$ is a left-evanescent wave.

The situation changes drastically with the introduction of damping. In contradistinction to the undamped case, when $\delta \neq 0$ the homogeneous differential equation no longer has pure propagating, i.e., constant-amplitude, longitudinal modes. Indeed, by Fourier transformin $;$ equation (1.3b) in the $r$ direction, we arrive at the family of ODEs

$$
\hat{E}_{z z}+\left(k_{0}^{2}(1+i \delta)-\lambda\right) \hat{E}=0
$$

parameterized by the dual variable $\lambda$. Each of the equations (3.9) has two linearly independent solutions:

$$
\begin{gathered}
\hat{E}_{1}=e^{i z} \sqrt{k_{c}^{2}+i k_{0}^{2} \delta}=e^{i k_{c} z \sqrt{1+i \frac{k_{i}^{2}}{k_{r}^{2} \delta}},} \\
\hat{E}_{2}=e^{-i z \sqrt{k_{r}^{2}+i k_{0}^{2} \delta}}=e^{-i k_{c} z \sqrt{1+i \frac{k_{0}^{2}}{k_{r}^{2}} \delta}} .
\end{gathered}
$$

Clearly, the second equality in each formula (3.10) is valid only if $k_{c} \neq 0$ Formulae (3.10) show that as long as $\delta \neq 0$ there will always be a nontrivial real part in each exponent. (onsequently, the amplitudes of the waves (3.10) will always decrease or increase exponentially for $z \longrightarrow \pm \infty$. In particular, if we analyze the traveling waves regime of the undamped equation, i.e., the case of small $\lambda: k_{0}^{2}-\lambda>0$, and additionally assume that $|\delta| \ll 1$, then formulae (3.10) yield (cf. formulae (3.6)):

$$
\begin{aligned}
& \hat{E}_{1}^{\text {(damped) }} \approx e^{i k_{r}=\left(1+i \frac{1}{2} \frac{k_{0}^{2}}{k_{c}^{2} \delta}\right)}=e^{i k_{r} z-\frac{1}{2} \frac{k_{0}^{2}}{k_{c}} \delta z}=\hat{E}_{1}^{\text {(undamped) }} \cdot e^{-\frac{1}{2} \frac{k_{2}^{2}}{k_{c}} \delta z} \\
& \hat{E}_{2}^{\text {(damped) }} \approx e^{-i k_{r}=\left(1+i \frac{1}{2} \frac{k_{0}^{2}}{k_{r}^{2}} \delta\right)}=e^{-i k_{r} z+\frac{1}{2} \frac{k_{0}^{2}}{k_{c}} \delta z}=\hat{E}_{2}^{\text {(un lamped) }} \cdot e^{\frac{1}{2} \frac{k_{0}^{2}}{k_{c}} \delta z} .
\end{aligned}
$$

Since we identify $\hat{E}_{1}^{\text {(undamped) }}=e^{i k_{c} z}$ of $(3.6)$ as the right-traveling wave, and $\hat{E}_{2}^{\text {(undamped) }}=e^{-i k_{c}=}$ of (3.6) as the left traveling wave, we can conclude that to have the propagation toward infinity (i.e., the radiation of waves) accompanied by the decay of the amplitude (as opposed to growth with no bound), we have to take positive values of the damping factor: $\delta>0$ (cf. Section 1). In this case, the amplitude of $\hat{E}_{1}^{\text {(damped) }}$ will decay exponentially for $z \longrightarrow+\infty$ (propagation to the right), and the amplitude of $\hat{E}_{2}^{\text {(damped) }}$ will decay exponentially for $z \longrightarrow-\infty$ (propagation to the left). As one can easily see from (3.11), the rate of decay is controlled by the value of $\delta$. 
In connection to the aforementioned exponential behavior of the longitudinal modes, a more general fact is also worth mentioning. The full Fourier symbol of the undamped operator of (1.3a) obviously has real roots on the dual plane, these roots occupy the entire circle of radius $k_{0}$ centered at the origin. In contradistinction to that, the symbol of the damped operator of $(1.3 \mathrm{~b})$ does not have real roots on the dual plane. As shown in [20], the damped operator will therefore have an exponentially decaying fundamental solution. In practical terms it means that the outgoing waves governed by the damped Helmholtz equation will decay exponentially toward infinity in all directions. For comparison we remind that the fundamental solution of the undamped operator is given by a zero order Hankel function, which only decays at infinity as the inverse square root of the distance from the origin.

To establish the properties of the propagating modes for the discretization (3.2) in the presence of damping, and to demonstrate similarities to the continuous damped case, we first introduce and prove

Proposition 3.1. The characteristic equation (3.3) for $\delta \neq 0$ does not have roots with unit magnitude.

Proof. Let us assume the opposite: There exists a unit magnitude root $q=e^{i \theta}$ to the algebraic characteristic equation (3.3). Then,

$$
\begin{aligned}
& -1+16 e^{i \theta}+\left(12 h_{z}^{2}\left(k_{0}^{2}(1+i \delta)-\lambda_{m}\right)-30\right) e^{2 i \theta}+16 e^{3 i \theta}-e^{4 i \theta} \\
= & {\left[-e^{-2 i \theta}+16 e^{-i \theta}+\left(12 h_{z}^{2}\left(k_{0}^{2}(1+i \delta)-\lambda_{m}\right)-30\right)+16 e^{i \theta}-e^{2 i \theta}\right] \cdot e^{2 i \theta} } \\
= & {\left[-2 \cos (2 \theta)+32 \cos \theta+\left(12 h_{z}^{2}\left(k_{0}^{2}(1+i \delta)-\lambda_{m}\right)-30\right)\right] \cdot e^{2 i \theta}=0 . }
\end{aligned}
$$

As $e^{2 i \theta} \neq 0$, the expression in rectangular brackets has to be equal to zero. Since the only imaginary contribution to this expression is proportional to $\delta$, we conclude that it is only possible when $\delta=0$.

Proposition 3.1 implies that similarly to the continuous case, there will be no constant-amplitude solutions to the homogeneous counterpart of the discrete equation (3.2). Each of the four corresponding modes: $q_{1}^{n}, q_{1}^{-n}, q_{2}^{n}$, and $q_{2}^{-n}$, will exponentially decrease in one direction and exponentially increase in the opposite direction. In particular, if we assume as before that $\alpha \ll 1$ in the undamped traveling waves regime, ${ }^{4}$ and in addition let $\delta \ll 1$, then solving first equations (3.4) for $d_{1}$, then equation (3.5a) for $q_{1}$ and $q_{1}^{-1}$, and finally using the Taylor expansion, we obtain (cf. formula (3.8))

$$
\begin{aligned}
q_{1} & =e^{i k_{c} h_{z}-\frac{1}{2} \frac{k_{0}^{2}}{k_{c}} \delta h_{z}}+\mathcal{O}\left(\left[k_{c} h_{z}\left(1+i \frac{1}{2} \frac{k_{0}^{2}}{k_{c}^{2}} \delta\right)\right]^{5}\right), \\
q_{1}^{-1} & =e^{-i k_{c} h_{z}+\frac{1}{2} \frac{k_{0}^{2}}{k_{c}} \delta h_{z}}+\mathcal{O}\left(\left[k_{c} h_{z}\left(1+i \frac{1}{2} \frac{k_{0}^{2}}{k_{c}^{2}} \delta\right)\right]^{5}\right) .
\end{aligned}
$$

Equalities (3.12) mean that the damped discrete traveling waves $q_{1}^{n}$ and $q_{1}^{-n}$ approximate the damped continuous waves (3.11) with the fourth order of accuracy. This result is obviously similar to the one obtained in the undamped case, see formulae (3.8).

As of yet, our discussion has focused on the first pair of roots $q_{1}$ and $q_{1}^{-1}$ of the characteristic equation (3.3), because these roots correspond to the genuine modes of the original differential equation. The second pair of roots $q_{2}$ and $q_{2}^{-1}$ is obtained by solving equations (3.4) for $d_{2}$ and subsequently solving equation (3.5b). The corresponding pair of solutions $q_{2}^{n}$ and $q_{2}^{-n}$ is, of course, a pure numerical artifact. In [12] we have shown that for $\delta=0$ the roots $q_{1}$ and $q_{1}^{-1}$ cannot have unit magnitude: $\left|q_{2}\right|<1$ and $\left|q_{2}^{-1}\right|>1$, which means that the waves $q_{2}^{n}$ and $q_{2}^{-n}$ are always evanescent. In the damped case, Proposition 3.1 implies that

\footnotetext{
${ }^{4}$ This would also imply $\frac{k_{0}^{2}}{k_{c}} h_{z} \ll 1$ because $\lambda_{m}$ is small and $k_{c} \sim k_{0}$.
} 
these waves will remain evanescent as well. The presence of the second pair of waves, however, implies that the discrete equation requires two more boundary conditions compared 10 the original differential equation.

In Section 1, we have outlined a general two-fold motivation behind the introduction of damping into the Helmholtz equation. One part was coming from physics because absorption by the medium always accompanies the propagation of electromagnetic waves in real-life sottings. Moreover, from the standpoint of mathematics the introduction of damping helps select a unique solution using the limiting absorption principle. Besides these two key reasons, the presence of damping in the equation also affects positively the properties of the numerical algorithm.

First, having no roots of unit magnitude presents a significant advantage from the vieupoint of numerical stability. In this case, every discrete system (3.2) supplemented by the boundary conditions that are discussed below in Section 3.3, will be well-posed in the classical sense of [13.21]. In contradistinction to that, in the original undamped case existence of the roots with unit magnitude may, generally speaking. cause a weak polynomial growth of the error when the grid size is refined, although n m major exponential instability will be possible.

Second, we remind that the original formulation of the problem requi es that $E(z, r)$ vanish as $|r| \longrightarrow \infty$. Instead, when solving the problem numerically we set $E(z, r)=0$ at a lar ge but still finite distance $r=r_{\text {max }}$. Of course, we expect that on some fixed bounded region of interest located next to the axis of the propagating beam our solution will converge to the original infinite-domain solution with the increase of $r_{\max }$. A general methodology for solving infinite-domain problems based on a similar idra was first introduced and studied in $[22,23,25,26]$ in the context of fluid flow. It was shown, in particular, that one may obtain the convergence

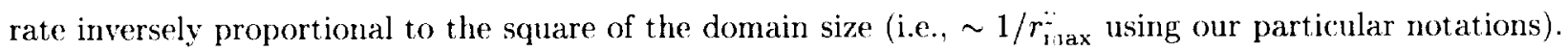
Besides, for a specific example that involves the Laplace equation that transforms into a Yukawa equation by introducing small "dissipation," Mishkov and Ryaben'kii have shown in [18] that one may expect a much faster convergence of the damped solution to the undamped one on a fixed-size domain rather than on the original unbounded domain. Even though the formulation of the problem in [18] is not quite the same as the one analyzed here, there are still similarities that allow us to consider the results of [18] as another argument toward using the damped equation.

3.3. Boundary Conditions. Apart from the foregoing key difference in the properties of the roots of equation (3.3) in the undamped and damped case, see Section 3.2, the alg orithm for solving the damped NLH remains basically the same as the undamped algorithm of [12]. Each equation (3.2) needs to be supplemented by the radiation boundary conditions at $z=z_{\max }$ and two-way $\mathrm{ABC}$ 's at $z=0$.

The radiation boundary conditions are constructed by requiring that on the right boundary $z=z_{\max }$ the solution of (3.2) be composed of only the waves that propagate/decay to the right, i.e., $\hat{E}_{n}=c_{1} q_{1}^{n}+c_{2} q_{2}^{n}$. The selection is rendered by the so-called one-way discrete Helmholtz equation [12], which is a a linear homogeneous relation that defines the span of all the appropriate modes. Specifically, let us consider equation (3.2) on the grid $n=0,1, \ldots, N-1, N$, and assume that the right-hand side $\hat{g}_{n}$ is small and can therefore be neglected near the right boundary $n=N$, i.e., that the propagaticn is almost linear in the far field. Then, we require that the vector $\left[\hat{E}_{N-3}, \hat{E}_{N-2}, \hat{E}_{N-1}, \hat{E}_{N}\right]^{T}$ be a linear combination of the two vectors: $\left[q_{1}^{N-3}, q_{1}^{N-2}, q_{1}^{N-1}, q_{1}^{N}\right]^{T}$ and $\left[q_{2}^{N-3}, q_{2}^{N-2}, q_{2}^{N-1}, q_{2}^{N}\right]^{T}$, which obviously translates into

$$
\operatorname{Rank}\left[\begin{array}{cccc}
\hat{E}_{N-3} & \hat{E}_{N-2} & \hat{E}_{N-1} & \hat{E}_{\Lambda} \\
1 & q_{1} & q_{1}^{2} & q_{1}^{3} \\
1 & q_{2} & q_{2}^{2} & q_{2}^{3}
\end{array}\right]=: 2 .
$$


Relation (3.13) is, in turn, equivalent to the two scalar equalities

$$
\begin{gathered}
q_{1} q_{2} \hat{E}_{N-3}-\left(q_{1}+q_{2}\right) \hat{E}_{N-2}+\hat{E}_{N-1}=0, \\
q_{1} q_{2} \hat{E}_{N-2}-\left(q_{1}+q_{2}\right) \hat{E}_{N-1}+\hat{E}_{N}=0,
\end{gathered}
$$

which constitute the one-way-to-the-right discrete Helmholtz equation. Relations (3.14a) and (3.14b) supplement the scheme (3.2) at $n=N-1$ and $n=N$, respectively, i.e., at the two near-edge nodes of the grid where the regular five-point wide stencil of (3.2) cannot be applied.

The two-way $A B C$ at $z=0$ also has to possess the capability of radiation boundary conditions, i.e., it has guarantee the transparency of the interface for all the waves that propagate/decay to the left. In other words, we require that at the left boundary the outgoing, i.e., scattered, waves be given by $\hat{E}_{n}^{\text {(scat) }}=c_{1} q_{1}^{-n}+c_{2} q_{2}^{-n}$. Assuming for a second the homogeneity: $\hat{g}_{n}=0$ near $n=0$, we could obtain similarly to (3.13):

$$
\operatorname{Rank}\left[\begin{array}{cccc}
\hat{E}_{0}^{\text {(scat })} & \hat{E}_{1}^{\text {(scat })} & \hat{E}_{2}^{\text {(scat })} & \hat{E}_{3}^{\text {(scat) }} \\
1 & q_{1}^{-1} & q_{1}^{-2} & q_{1}^{-3} \\
1 & q_{2}^{-1} & q_{2}^{-2} & q_{2}^{-3}
\end{array}\right]=2 .
$$

Relation (3.15), again, is equivalent to the one-way-to-the-left discrete Helmholtz equation:

$$
\begin{aligned}
& \hat{E}_{0}^{\text {(scat) }}-\left(q_{1}+q_{2}\right) \hat{E}_{1}^{\text {(scat) }}+q_{1} q_{2} \hat{E}_{2}^{\text {(scat) }}=0, \\
& \hat{E}_{1}^{\text {(scat) }}-\left(q_{1}+q_{2}\right) \hat{E}_{2}^{\text {(scat) }}+q_{1} q_{2} \hat{E}_{3}^{\text {(scat })}=0 .
\end{aligned}
$$

Equations (3.16a), (3.16b), however, cannot be immediately used as the $\mathrm{ABC}$ at $z=0$ because the foregoing assumption of homogeneity near the interface is, generally speaking, not correct, and moreover, equations (3.16a), (3.16b) do not account for the incoming wave at $z=0$ (see Section 2.1), i.e., do not have the important two-way capability. The analysis of [12] shows that to accurately address both issues, i.e., the inhomogeneity that comes from the previous iteration and the presence of the incoming wave, it is sufficient to introduce particular modifications to the right-hand side $g_{n}$ only at two nodes: $n=0$ and $n=1$. The corresponding modification due to the incoming signal is obtained by simply substituting the right-traveling incoming wave $\hat{E}_{0}^{(i n c)} q_{1}^{n}$ into the one-way-to-the-left Helmholtz equation (3.16a), (3.16b). Altogether, the two-way $\mathrm{ABCs}$ at $z=0$ are given by (cf. formulae (3.16a), (3.16b)):

$$
\begin{aligned}
& \hat{E}_{0}-\left(q_{1}+q_{2}\right) \hat{E}_{1}+q_{1} q_{2} \hat{E}_{2}=\hat{g}_{0}^{\prime}, \\
& \hat{E}_{1}-\left(q_{1}+q_{2}\right) \hat{E}_{2}+q_{1} q_{2} \hat{E}_{3}=\hat{g}_{1}^{\prime},
\end{aligned}
$$

where prime denotes the aforementioned modification of the right-hand side, see [12]. Again, relations (3.17a) and $(3.17 \mathrm{~b})$ supplement the scheme (3.2) at the near-edge nodes $n=0$ and $n=1$, respectively, where the regular five-point stencil cannot be applied. Straightforward considerations based on the linear superposition principle and uniqueness (see [12]) guarantee that inhomogeneous relations (3.17a), (3.17b) correctly specify the incoming signal at $z=0$ and still ensure the reflectionless propagation of all the outgoing waves through $z=0$ toward $z=-\infty$.

3.4. Computational Complexity. The computational complexity of one solution of equation (3.1) is $\mathcal{O}\left(N_{z} N_{r} \ln N_{r}\right)$ operations, where $N_{z}$ and $N_{r}$ are the corresponding grid dimensions. Indeed, the cost of solving each of the $N_{r}$ one-dimensional systems (3.2) is linear with respect to $N_{z}$, because each of this systems needs to be solved repeatedly for multiple right-hand sides. As such, the sparse LU decomposition can be performed only once ahead of time, and the cost of backward substitution is linear. Therefore, the overall complexity is dominated by the cost of $N_{z}$ direct and inverse FFT's of length $N_{r}$, which is $\mathcal{O}\left(N_{z} N_{r} \ln N_{r}\right)$. 
4. Results. In this section we present simulation results for the Gaussian initial conditions $E_{\text {inc }}^{0}=$ $\exp \left(-r^{2}\right)$ and $\psi_{0}=\left(\epsilon r_{0}^{2} k_{0}^{2}\right)^{1 / 4} \exp \left(-r^{2} / r_{0}^{2}\right)$ for the NLH and NLS, respectively. Denoting, as before, the input power of the incoming wave by $N(0)$, we define the fractional input power as

$$
p=N(0) / N_{c},
$$

i.e., $p=1$ when the input power is equal to the NLS critical power $N_{c}$. For the Gaussian initial conditions used in our simulations $p=k_{0} \sqrt{2 \epsilon / 3 \pi}$ [12]. In all simulations we set $k_{0}=8$ and $r_{0}=1$.

TABLE: 1.1

Threshold values of linear damping $\delta$.

\begin{tabular}{|c|c|c|c|c|}
\hline Case No. & $\epsilon$ & $p=N(0) / N_{\mathrm{c}}$ & $\delta_{\mathrm{th}}^{1 \mathrm{H}}$ & $\delta_{\mathrm{th}}^{\mathrm{S}}$ \\
\hline \hline 1 & 0.06 & $90 \%$ & 0 & 0 \\
\hline 2 & 0.07 & $97.5 \%$ & 0 & 0 \\
\hline 3 & 0.072165819 & $99 \%$ & 0 & 0 \\
\hline 4 & $3 \pi / 128$ & $100 \%$ & $9.6 \cdot 10$ & 0 \\
\hline 5 & 0.075 & $100.9 \%$ & 0.00023 & 0 \\
\hline 6 & 0.08 & $104 \%$ & 0.0007 & 0.00025 \\
\hline 7 & 0.1 & $116 \%$ & 0.0027 & 0.0025 \\
\hline 8 & 0.125 & $130 \%$ & 0.0049 & 0.0062 \\
\hline 9 & 0.15 & $142 \%$ & $0.0071)$ & 0.010 \\
\hline 10 & 0.2 & $164 \%$ & $0.014 ;$ & 0.019 \\
\hline 11 & 0.3 & $202 \%$ & 0.030 & 0.035 \\
\hline 12 & 0.4 & $233 \%$ & 0.044 & 0.050 \\
\hline 13 & 0.5 & $261 \%$ & $0.05 \%$ & 0.065 \\
\hline
\end{tabular}

In Table 4.1 we show the calculated threshold values $\delta_{\mathrm{th}}^{\mathrm{H}}$ and $\delta_{\mathrm{th}}^{\mathrm{S}}$. The quantity $\delta_{\mathrm{hh}}^{\mathrm{H}}$ in Table 4.1 represents the smallest non-negative value of $\delta$ for which we obtain a global solution of the NLH. By this we mean that the nonlinear iterations converge in the sense that the value of $\max _{z, r}\left(E^{(n+1)}-E^{(n)}\right) / \max _{z, r} E^{(n+1)}$ drops by at least a factor of $10^{-6}$ in the course of iterations on the computational domain $0 \leq z \leq 40$ and $0 \leq r \leq 40$, with grid sizes $h_{z}=\lambda / 20$ and $h_{r}=\lambda / 8$, where $\lambda=2 \pi / k_{0}$. The particular choice of the domain size and grid resolution is "inherited" from our previous numerical experiments, see [10,12]. The values of $\delta_{\mathrm{th}}^{\text {H }}$ in Table 4.1 are obtained with at least two significant digits by repeatedly running the code for a given $\epsilon$ and varying $\delta$, which allows one to "close in" on the threshold. As, however, liscussed in Section 1, with a larger computational domain and/or a finer grid it may be possible to obrain regular solutions for smaller values of $\delta$, hence, to obtain a lower value of the threshold $\delta_{\mathrm{th}}^{\mathrm{H}}$. For example, using the same computational domain and twice as fine grid: $h_{\tau}=\lambda / 40$ and $h_{r}=\lambda / 16$, we could obtain $\delta_{\mathrm{th}}^{\mathrm{H}}=0.0133$ instead of $\delta_{\mathrm{th}}^{\mathrm{H}}=0.0145$ for the data on row No. 10 of Table $4.1(f=0.2)$. Likewise, using the original grid resolution $h_{z}=\lambda / 20$ and $h_{r}=\lambda / 8$ and the computational domain which was twice as large: $z_{\max }=80$ and $r_{\max }=80$, we could obtain $\delta_{\mathrm{th}}^{\mathrm{H}}=0.0022$ instead of $\delta_{\mathrm{th}}^{\mathrm{H}}=0.0027$ for the data on row No. 7 of Table $4.1(\epsilon=0.1)$. In other words, the values of $\delta_{\mathrm{th}}^{\mathrm{H}}$ from Table 4.1 should be considered upper bounds for the actual thresholds. However, the quantitative limits of pursuing this venue are still unexplored, i.e., it is not known how far down in $\delta_{\text {th }}^{\mathrm{H}}$ one can go by increasing the domain size and/or grid resolution. Our ability to answer this question is obviously limited by computer resources, and as of yet the question remains open. In particular, it is unclear whether 
we can achieve $\delta_{\mathrm{th}}^{\mathrm{H}}=0 \mathrm{by}$ choosing a sufficiently large domain and/or fine grid.

Similarly, the quantity $\delta_{\mathrm{th}}^{\mathrm{S}}$ in Table 4.1 represents the smallest non-negative value of damping $\delta$ for which the NLS solution does not blow up. In our NLS simulations we use standard fourth-order finite difference schemes for the spatial derivatives and explicit fourth-order Runge-Kutta for marching in $z$. As has recently been shown in [9], in finite-difference simulations of NLS solutions that are known analytically to become singular, the computed solution still remains bounded. Therefore, there is always an element of arbitrariness in selecting a numerical criterion for blowup in NLS simulations. In our NLH simulations the largest relative increase in amplitude due to self-focusing has never exceeded a factor of two. In order to make the blowup criteria in NLH and NLS simulations as close to one another as possible, we define the computed NLS solution as becoming singular once its amplitude increases by a factor of two. We checked that altering this NLS blowup criterion leads to only minor changes in the results for $\delta_{\mathrm{th}}^{\mathrm{S}}$. For example, using the blowup) criterion of relative focusing by a factor of 4 , rather than 2, for $\epsilon=0.08$ (row No. 6 of Table 4.1) gives $\delta_{\mathrm{th}}^{\mathrm{S}}=0.00021$ instead of $\delta_{\mathrm{th}}^{\mathrm{S}}=0.00025$; and using this new criterion for $\epsilon=0.15$ (row No. 9 of Table 4.1) yields $\delta_{\mathrm{th}}^{\mathrm{S}}=0.0089$ instead of $\delta_{\mathrm{th}}^{\mathrm{S}}=0.010$. In particular, this change does not affect our main finding of initial conditions for which $\delta_{\mathrm{th}}^{\mathrm{H}}<\delta_{\mathrm{th}}^{\mathrm{S}}$.

As expected, for both the NLS and the NLH the threshold values of $\delta$ increase with $\epsilon$ (i.e., a larger amount of damping is needed to arrest collapse of beams with higher input power). For $\epsilon=0.06$ and $\epsilon=0.07$ the input power is below critical. Therefore, both the NLS and the NLH have global solutions for $\delta=0$. This behavior for the NLH holds (at least) till $\epsilon=0.072165819$, which corresponds to the last subcritical value ${ }^{5}$ that we have checked: $N(0)$ being equal to $99 \%$ of $N_{\mathrm{c}}$.

Starting from $\epsilon=3 \pi / 2 k_{0}^{2} \approx 0.073631077$, which corresponds exactly to $N(0)=N_{\mathrm{c}}$, the NLH requires a cortain positive amount of damping $\delta$ to maintain the regularity of the solution. For the NLS, the solution with no damping remains regular till $\epsilon=0.75$, which corresponds to $p=N(0) / N_{\mathrm{c}}=1.009$. Indeed, it is known that $N_{\mathrm{c}}$ is only a lower bound for the threshold power for NLS collapse, and that any initial condition which does blow up, and whose amplitude $\left|\psi_{0}\right|$ is not equal to the ground state profile $3^{1 / 4} \sqrt{\operatorname{sech}(2 r)}$, has power strictly above $N_{\mathrm{c}}[7,16,17]$. In our simulations we have discovered that for $\epsilon=3 \pi / 2 k_{0}^{2}$, which is the critical value for the NLH, as well as for the moderately supercritical values $\epsilon=0.075, \epsilon=0.08$ and $\epsilon=0.1$, when the input power $N(0)$ is only slightly above $N_{\mathrm{c}}$, the threshold damping for the NLH is larger ${ }^{6}$ than that for the NLS: $\delta_{\mathrm{th}}^{\mathrm{H}}>\delta_{\mathrm{th}}^{\mathrm{S}}$. However, for input powers that are equal or higher than $1.30 N_{\mathrm{c}}$ (which corresponds to $\epsilon=0.125)$ this trend reverses, see Table 4.1 , and we obtain $\delta_{\mathrm{th}}^{\mathrm{H}}<\delta_{\mathrm{th}}^{\mathrm{S}}$. Thus, for $N(0) \geq 1.30 N_{c},{ }^{7}$ there must be other mechanisms in the NLH not present in the NLS that help suppress the formation of singularity in the solution. Therefore, we may conclude that in this regime nonparaxiality and backscattering help arrest collapse of nonlinear waves.

In [6] Fibich has used asymptotic analysis to show that

$$
\delta_{\mathrm{th}}^{\mathrm{S}} \sim c(p-1)^{3 / 2}
$$

where $p$ is the fractional critical power (4.1). In Figure 4.1 we put this theoretical prediction to a test by plotting the values of $\delta_{\mathrm{th}}^{\mathrm{S}}$ and $\delta_{\mathrm{th}}^{\mathrm{H}}$ as a function of $(p-1)$. When we computed the best fit of the values of $\delta_{\mathrm{th}}^{\mathrm{S}}$ with the two-parameter family of curves $\delta_{\mathrm{th}}=c(p-1)^{\alpha}$, we obtained $\alpha=1.517$, which is in excellent

\footnotetext{
"As mentioned in Section 1, for larger subcritical values of $N(0)$ the convergence of nonlinear iterations becomes prohibitively slow.

${ }^{6}$ We remind that the values of $\delta_{\mathrm{th}}^{\mathrm{H}}$ in Table 4.1 are only upper bounds for the threshold; lower values may be obtained by refining the grid and/or enlarging the computational domain.

${ }^{7}$ More precisely, $N(0)$ higher than some value between $1.16 N_{c}$ and $1.30 N_{c}$
} 
agreement with formula (4.2). Relation (4.2) also provides a good approximation to the data points $\delta_{11}^{11}$, see Figure 4.1. The only exception is the lowest-power NLH data point in Figure 4.1 that corresponds to

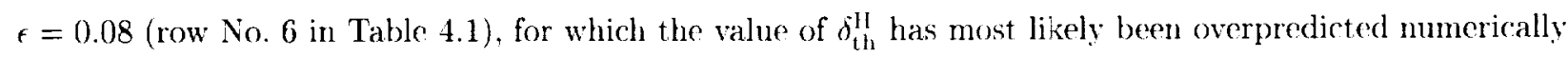
because of the computational constraints discussed previously.

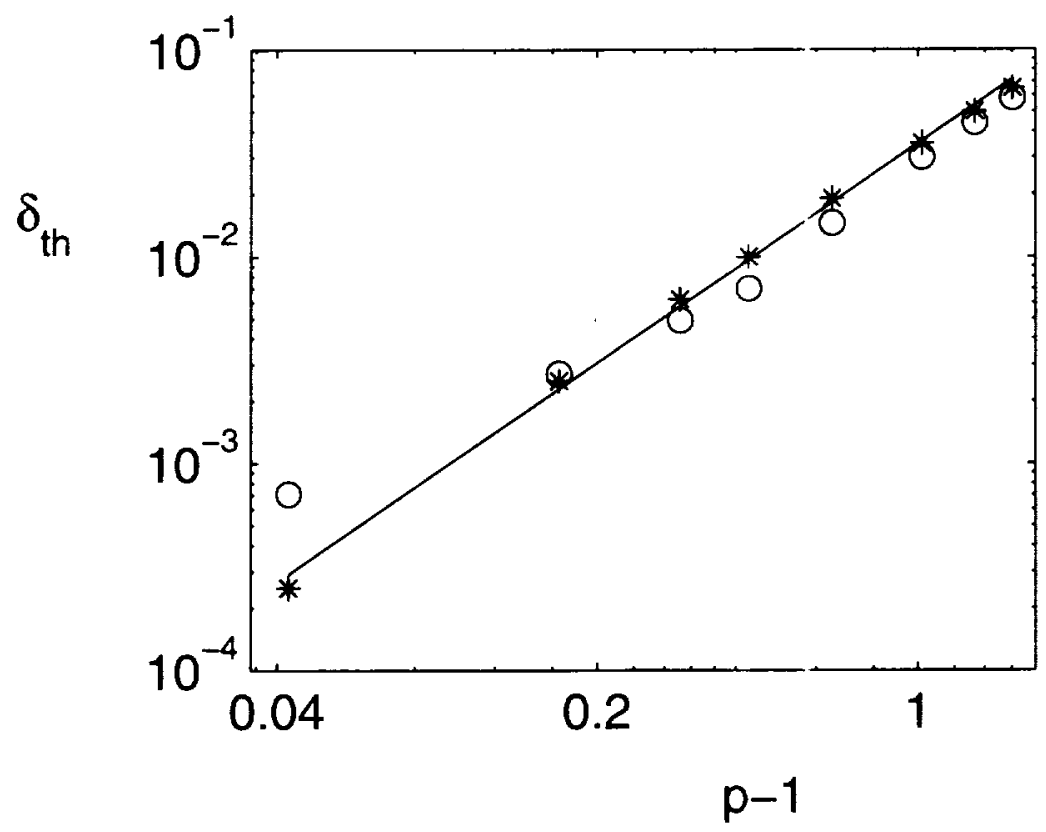

FI(. 4.1. Threshold values $\delta_{\mathrm{th}}^{\mathrm{H}}$ (hollow bullet.s "o") and $\delta_{\mathrm{th}}^{\mathrm{S}}$ (asterisks "*") as a fanction of ( $\left.p-1\right)$ for the data in Table 4.1 . The solid line $0.035(p-1)^{1.517}$ is the best fit to the values of $\delta_{\mathrm{th}}^{\mathrm{S}}$.

In Figure 4.2 we plot the on-axis $(r=0)$ amplitudes of the NLH and NLS solutions for $\epsilon=0.2$ and various values of $\delta$. The on-axis behavior is most representative of the phy sical processes that we are studying. because for symmetric beams this is the location of the peak intensity. When $\delta=\delta_{\mathrm{th}}^{\mathrm{H}}=0.0145$, the NLH solution exists globally but the NLS solution becomes singular at a finitc propagation distance. As the value of damping increases, both the NLS and the NLH solutions undergo less focusing. For all the cases for which both solutions remain regular, the NLS solution curve is higher than the NLH one from $z=0$ until its maximum, i.e., the point of the arrest of collapse. This provides additional support to the foregoing conclusion that nonparaxiality and backscattering arrest collapse of nonlinear waves. Note that after the collapse has been arrested, the NLS solution becomes lower than the NLS one. One possible explanation for this is that the NLS solution is undergoing higher focusing, hence it loses more power due to damping.

We emphasize that at $z=0$ the NLH solution is not equal to $E_{\mathrm{inc}}^{0}$, see Figure 4.2. The difference between the two is due to backscattering, and can be used to quantify the level of backscattering for a particular setting, see $[10,12]{ }^{8}$ In Table 4.2 we provide the values of miximum self-focusing and maximum backscattering in the NLH, defined as $\max _{r, z}|E(z, r)|$ and $\max _{r}\left|E(0, r)-E_{\mathrm{inc}}^{0}(r)\right|$, respectively, for various values of $\epsilon$ and $\delta$. The dash "...." in a particular cell of Table $4.2 \mathrm{~m} \epsilon$ ans that the level of damping was

${ }^{R}$ There are, in fact, two phenomena that account for the discrepancy between the NLH and NLS curves: Nonparaxiality of the forward propagating wave and backscattering. Because the problem is nonlinear. these two mechanisms cannot be easily and explicitly told apart inside the domain. The only location where we can clearly say that the difference is purely due to backscattering is the "inflow" interface $z=0$, see [10]. 

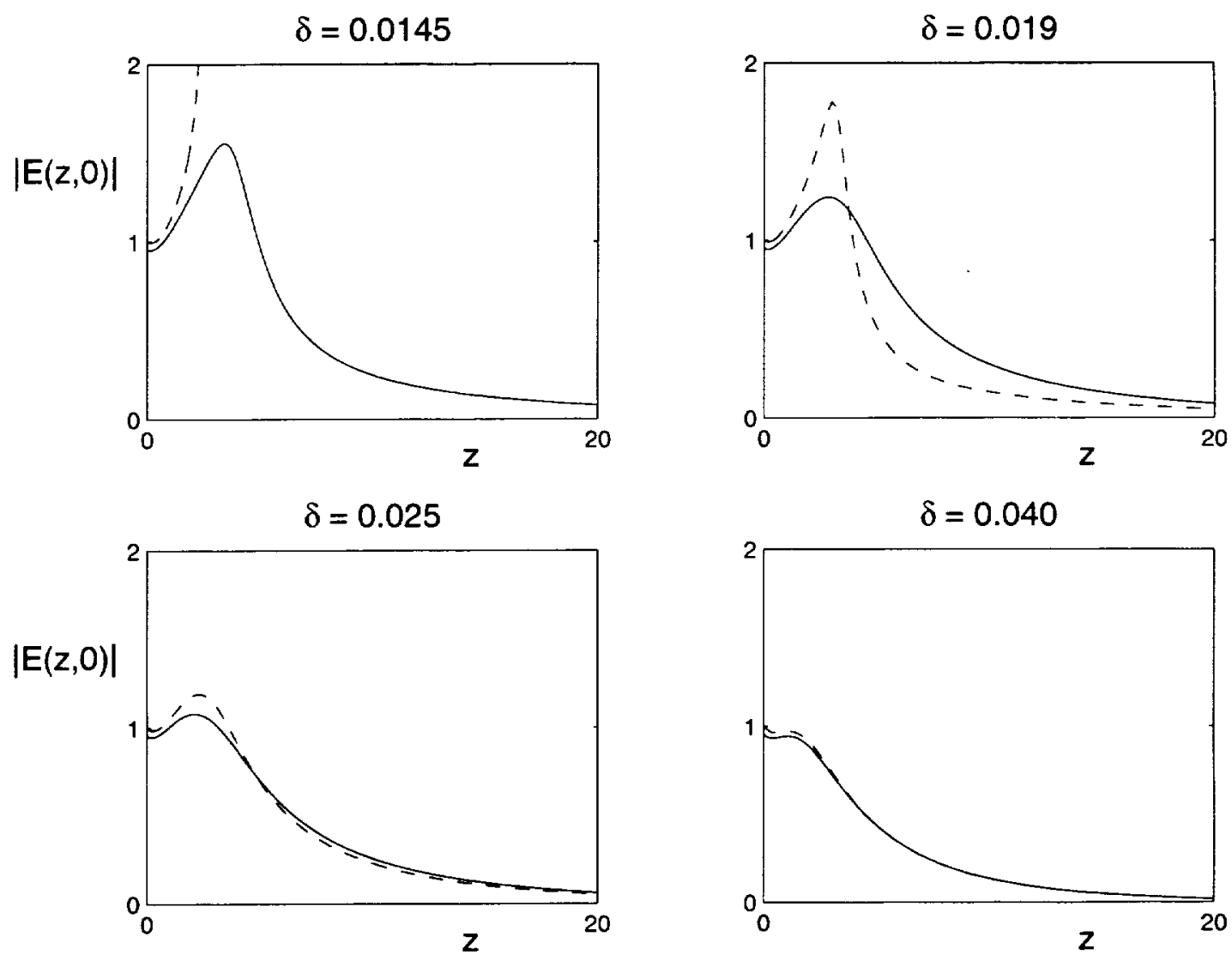

FIG. 4.2. On-axis amplitude of NLH (solid) and NLS (dashes) solutions for $\epsilon=0.2$ and various values of $\delta$.

insufficient to guarantee the convergence of the numerical algorithm. As expected, for a given level of damping $\delta$, the NLH solution undergoes stronger self-focusing as the nonlinearity coefficient $\epsilon$ increases. The level of backscattering also increases with the increase of $\epsilon$. As also expected, for a given input power $\epsilon$, when the damping $\delta$ increases the NLH solution undergoes weaker self-focusing (see Figure 4.2). Surprisingly, however, changing the value of damping $\delta$ has very little or no effect on the level of backscattering. To further corroborate this observation, we picked a particular value of the nonlinearity coefficient, $\epsilon=0.2$, and ran an additional series of numerical tests with a substantially more fine sampling for $\delta$. These results, which are presented in Table 4.3, confirm that backscattering is not affected by linear damping. This phenomenon certainly cannot be explained by saying that linear damping has the overall negligible effect, since its effect

TABLE 4.2

Maximum absolute levels of self-focusing and backscattering in the NLH for a variety of $\epsilon$ and $\delta$.

\begin{tabular}{|l|c|c|c||c|c|c|}
\hline & \multicolumn{3}{|c||}{ Maximum self-focusing } & \multicolumn{3}{c|}{ Maximum backscattering } \\
\cline { 2 - 7 } & $\delta=0.0145$ & $\delta=0.0175$ & $\delta=0.0210$ & $\delta=0.0145$ & $\delta=0.0175$ & $\delta=0.0210$ \\
\hline$\epsilon=0.15$ & 1.1179 & 1.0601 & 1.0162 & 0.0372 & 0.0373 & 0.0373 \\
\hline$\epsilon=0.175$ & 1.2718 & 1.1538 & 1.0761 & 0.0420 & 0.0421 & 0.0421 \\
\hline$\epsilon=0.2$ & 1.5515 & 1.3158 & 1.1716 & 0.0465 & 0.0466 & 0.0466 \\
\hline$\epsilon=0.225$ & - & - & 1.3242 & - & - & 0.0509 \\
\hline
\end{tabular}


on the focusing dynamics can be clearly seen through the changing values of the maximum focusing both in Table 4.2 and in Figure 4.2. At present, we have no good explanation to this surprising obserration.

TAHLF 4.3

Maximum absolute levels of self-focusing and backscattering in the NLH for $\mathrm{t}=0.2$.

\begin{tabular}{|c|c|c|c|}
\hline Case No. & Damping $\delta$ & Max. self-focusing & Max. backscattering \\
\hline \hline 1 & 0.0145 & 1.5515 & 0.0465 \\
\hline 2 & 0.0147 & 1.5296 & 0.0465 \\
\hline 3 & 0.0150 & 1.4992 & 0.0465 \\
\hline 4 & 0.0155 & 1.4538 & 0.0465 \\
\hline 5 & 0.0160 & 1.4135 & 0.0466 \\
\hline 6 & 0.0165 & 1.3776 & 0.0466 \\
\hline 7 & 0.0170 & 1.3451 & 0.0466 \\
\hline 8 & 0.0175 & 1.3158 & 0.0466 \\
\hline 9 & 0.0180 & 1.2892 & 0.0466 \\
\hline 10 & 0.0190 & 1.2428 & 0.0466 \\
\hline 11 & 0.0200 & 1.2041 & 0.0466 \\
\hline 12 & 0.0210 & 1.1716 & 0.0466 \\
\hline
\end{tabular}

5. Concluding Remarks. The question whether nonparaxiality and backscattering may arrest collapse of nonlinear waves has been open for many years. While the answer to this question is probably positive, no conclusive argument toward it, whether analytical or numerical, has been previously available in the literature. In this study we addressed this question within the framework of the linearly damped NLH and NLS. As has been mentioned. the addition of linear damping is not ad-hoc because it has both physical and mathematical motivation. Methodologically, linear damping provicles a very useful "extra dimension" that allowed us to efficiently control the solutions of the NLH and NLS. Specifically, the variation along this extra dimension has helped us to numerically identify the regimes, for which the NLS solution blows up, while the NLH solution remains regular. In other words, our results fursish the first ever definite numerical evidence that nonparaxiality and backscattering can arrest collapse. The question whether regular solutions to the NLH still exist in the absence of damping remains open as of yot. However, we hope that the arguments based on linear damping and the limiting absorption principle may be useful for proving global existence and uniqueness, both for the damped NLH and for the undamped NLH

\section{REFERENCES}

[1] N. Akhmediev, A. Ankiewicz, And J. M. Soto-Crespo, Does the nonlinear Schrödinger equation correctly describe beam propagation?, Opt. Lett., 18 (1993). pp. 411413.

[2] N. AKhmedev and J. M. SoTo-Crespo, Generation of a train of three-dimensional optical solitons in a self-focusing medium, Phys. Rev. A, 47 (1993), pp. 13581364.

[3] R. W. BoYd, Nonlinear Optics, Academic Press, Boston, 1992.

[4] M. D. FFIT AND .J. A. FLECK, Beam nonparaxiality, filament formation, and beam breakup in the self-focusing of optical beams, J. Opt. Soc. Am. B, 5 (1988). pp. 633640. 
[5] G. FiBıcil, Small beam nonparaxiality arrests self-focusing of optical beams, Phys. Rev. Lett., 76 (1996), pp. $4356-4359$.

[6] - Self-focusing in the damped nonlinear Schrödinger equation, SIAM. J. Appl. Math, 61 (2001). pp. $1680-1705$.

[7] G. FIBICh AND A. GaETA, Critical power for self-focusing in bulk media and in hollow waveguides, Opt. Lett., 25 (2000), pp. 335-337.

[8] G. FIBICH AND B. ILAN, Self focusing of elliptic beams: An example of the failure of the aberrationless approximation, JOSA B, 17 (2000), pp. 17491758.

[9] —. Discretization effects in the nonlinear Schrödinger equation, Appl. Numer. Math., to appear, (2003).

[10] G. Fibich, B. ILAN, AND S. V. Tsynkov, Computation of nonlinear backscattering using a high-order numerical method, J. Sci. Comput., 17 (2002), pp. 351-364.

[11] G. Fibich and G. C. Papanicolaot, Self-focusing in the perturbed and unperturbed nonlinear Schrödinger equation in critical dimen.sion, SIAM J. Appl. Math., 60 (1999), pp. 183240.

[12] G. Fibich and S. V. Tsynkov, High-order two-way artificial boundary conditions for nonlinear wave propagation with backscattering, J. Comput. Phys., 171 (2001), pp. 632 677.

[13] S. K. Godunov AND V. S. RYaben'KII, Canonical forms of systems of ordinary linear differential equations with constant coefficients, U.S.S.R. Comput. Math. and Math. Phys., 3 (1963), pp. 281 295.

[14] J. D. JACKson, Classical Electrodynamics, Wiley, New-York, 1975.

[15] P. L. Kelley, Self-focusing of optical beams, Phys. Rev. Lett., 15 (1965), pp. 1005 1008.

[16] F. MFrle, On uniqueness and continuation properties after blow-up time of self-similar solutions of nonlinear Schrödinger equation with critical exponent and critical mass, Comm. Pure Appl. Math., 45 (1992), pp. 203254.

[17] - Determination of blow-up solutions with minimal mass for nonlinear Schrödinger equation with critical power, Duke Math. J., 69 (1993), pp. 427-454.

[18] M. N. Mishkov and V. S. RYaben'KII, Investigation of artificial boundary conditions constructed by periodization and the introduction of a small parameter for subsonic fiow problems, Mathematical Modeling, 10 (1998), pp. 87-98. (In Russian).

[19] A. C. Newell and J. V. Moloney, Nonlinear Optics, Addison-Wesley, Redwood City, Calif., 1992.

[20] V. P. PaLAmodov, Conditions at infinity for correct solvability of a certain class of equations of the form $p\left(i \frac{\partial}{\partial x}\right) u=f$, Dokl. Akad. Nauk SSSR, 129 (1959), pp. 740-743.

[21] V. S. RYABEN'KIl, Necessary and sufficient conditions for good definition of boundary value problems for systems of ordinary difference equations, U.S.S.R. Comput. Math. and Math. Phys., 4 (1964), pp. $43-61$.

[22] V. S. RYABEN'kil and S. V. Tsynkov, Artificial boundary conditions for the numerical solution of external viscous flow problems, SIAM J. Numer. Anal., 32 (1995), pp. 1355-1389.

[23] S. V. Tsynkov, An application of nonlocal external conditions to viscous flow computations, J. Comput. Phys., 116 (1995), pp. 212-225.

[24] - Numerical solution of problems on unbounded domains. A review, Appl. Numer. Math., 27 (1998), pp. 465-532.

[25] - External boundary conditions for three-dimensional problems of computational aerodynamics, SIAM J. Sci. Comp., 21 (1999), pp. 166206. 
[26] S. V. Tsynkov, E. Turkel, and S. Abarbanel, External flow computations using global boundary conditions, AIAA Journal, 34 (1996), pp. 700706.

[27] V. S. Vladimirov, Equations of Mathematical Physics, Dekker, New-York, 1971.

[28] M. I. WEINSTEIN, Nonlinear Schrödinger equations and sharp int rpolation estimates, Comm. Math. Phys., 87 (1983), pp. 567-576. 


\begin{tabular}{|c|c|c|c|}
\hline \multicolumn{3}{|c|}{ REPORT DOCUMENTATION PAGE } & $\begin{array}{l}\text { Form Approved } \\
\text { OMB No. 0704-0188 }\end{array}$ \\
\hline \multicolumn{4}{|c|}{ 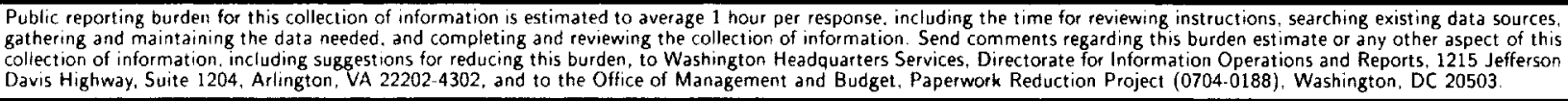 } \\
\hline 1. AGENCY USE ONLY (Leave blank) & $\begin{array}{l}\text { 2. REPORT DATE } \\
\text { September } 2002\end{array}$ & \multicolumn{2}{|c|}{$\begin{array}{l}\text { 3. REPORT TYPE AND DATES COVERED } \\
\text { Contractor Report }\end{array}$} \\
\hline \multicolumn{3}{|c|}{$\begin{array}{l}\text { 4. TITLE AND SUBTITLE } \\
\text { BACKSCATTERING AND NONPARAXIALITY ARREST } \\
\text { COLLAPSE OF DAMPED NONLINEAR WAVES }\end{array}$} & \multirow[t]{2}{*}{$\begin{array}{l}\text { 5. FUNDING NUMBERS } \\
\text { C NAS1-97046 } \\
\text { WU } 505-90-52-01\end{array}$} \\
\hline \multicolumn{3}{|c|}{$\begin{array}{l}\text { 6. AUTHOR(S) } \\
\text { G. Fibich, B. Ilan, and S. Tsynkov }\end{array}$} & \\
\hline \multicolumn{2}{|c|}{$\begin{array}{l}\text { 7. PERFORMING ORGANIZATION NAME(S) AND ADDRESS(ES) } \\
\text { ICASE } \\
\text { Mail Stop } 132 \mathrm{C} \\
\text { NASA Langley Research Center } \\
\text { Hampton, VA } 23681-2199\end{array}$} & & $\begin{array}{l}\text { 8. PERFORMING ORGANIZATION } \\
\text { REPORT NUMBER } \\
\text { ICASE Report No. } 2002-30\end{array}$ \\
\hline \multicolumn{3}{|c|}{$\begin{array}{l}\text { 9. SPONSORING/MONITORING AGENCY NAME(S) AND ADDRESS(ES) } \\
\text { National Aeronautics and Space Administration } \\
\text { Langley Research Center } \\
\text { Hampton, VA } 23681-2199\end{array}$} & $\begin{array}{l}\text { 10. SPONSORING/MONITORING } \\
\text { AGENCY REPORT NUMBER } \\
\text { NASA/CR-2002-211764 } \\
\text { ICASE Report No. } 2002-30\end{array}$ \\
\hline \multicolumn{4}{|c|}{$\begin{array}{l}\text { 11. SUPPLEMENTARY NOTES } \\
\text { Langley Technical Monitor: Dennis M. Bushnell } \\
\text { Final Report } \\
\text { To be submitted to the SIAM Journal on Applied Mathenatics. }\end{array}$} \\
\hline \multicolumn{2}{|c|}{$\begin{array}{l}\text { 12a. DISTRIBUTION/AVAILABILITY STATEMENT } \\
\text { Unclassified-Unlimited } \\
\text { Subject Category } 64 \\
\text { Distribution: Nonstandard } \\
\text { Availability: NASA-CASI (301) 621-0390 }\end{array}$} & & 12b. DISTRIBUTION CODE \\
\hline \multicolumn{4}{|c|}{$\begin{array}{l}\text { 13. ABSTRACT (Maximum } 200 \text { words) } \\
\text { The critical nonlinear Schrödinger equation (NLS) models the propagation of intense laser light in Kerr media. This } \\
\text { equation is derived from the more comprehensive nonlinear Helmholtz equation (NLH) by employing the paraxial } \\
\text { approximation and neglecting the backscattered waves. It is known that if the input power of the laser beam (i.e., } \\
\mathrm{L}_{2} \text { norm of the initial solution) is sufticiently high, then the NLS model predicts that the beam will self-focus to a } \\
\text { point (i.e., collapse) at a finite propagation distance. Mathematically, this behavior corresponds to the formation of } \\
\text { a singularity in the solution of the NLS. A key question which has been open for many years is whether the solution } \\
\text { to the NLH, i.e., the "parent" equation, may nonetheless exist and remain regular everywhere, in particular for those } \\
\text { initial conditions (input powers) that lead to blowup in the NLS. In the current study we address this question by } \\
\text { introducing linear damping into both models and subsequently comparing the numerical solutions of the damped } \\
\text { NLH (boundary-value problem) with the corresponding solutions of the damped NLS (initial-value problem). Linear } \\
\text { damping is introduced in much the same way as done when analyzing the classical constant-coefficient Helmholtz } \\
\text { equation using the limiting absorption principle. Numerically, we have found that it provides a very efficient tool for } \\
\text { controlling the solutions of both the NLH and NLS. In particular, we have been able to identify initial conditions } \\
\text { for which the NLS solution does become singular, whereas the NLH solution still remains regular everywhere. We } \\
\text { believe that our finding of a larger domain of existence for the NLH than that for the NLS is accounted for by } \\
\text { preciscly those mechanisms that have been neglected when deriving the NLS from the NLH, i.e., nonparaxiality and } \\
\text { backscattering. }\end{array}$} \\
\hline \multirow{2}{*}{\multicolumn{3}{|c|}{$\begin{array}{l}\text { 14. SUBJECT TERMS } \\
\text { Kerr medium, nonlinear wave propagation, self-focusing, singularity formation, } \\
\text { linear damping, limiting absorption, two-way } \mathrm{ABCs}\end{array}$}} & $\begin{array}{l}\text { 15. NUMBER OF PAGES } \\
24 \\
\end{array}$ \\
\hline & & & $\begin{array}{c}\text { 16. PRICE CODE } \\
\text { A03 }\end{array}$ \\
\hline $\begin{array}{l}\text { 17. SECURITY CLASSIFICATION } \\
\text { OF REPORT } \\
\text { Unclassified }\end{array}$ & $\begin{array}{l}\text { 18. SECURITY CLASSIFICATION } \\
\text { OF THIS PAGE } \\
\text { Unclassified }\end{array}$ & $\begin{array}{l}\text { 19. SECURITY CLASSIFICATION } \\
\text { OF ABSTRACT }\end{array}$ & $\begin{array}{l}\text { 20. LIMITATION } \\
\text { OF ABSTRACT }\end{array}$ \\
\hline NSN 7540-01-280-5500 & & & $\begin{array}{l}\text { Standard Form 298(Rev. 2-89) } \\
\text { Prescribed by ANSI Std. Z39-18 } \\
298-102\end{array}$ \\
\hline
\end{tabular}

